\title{
Prognostic value of NEK2 overexpression in digestive system cancers: a meta-analysis and systematic review
}

This article was published in the following Dove Press journal:

OncoTargets and Therapy

\author{
Qian Ren ${ }^{1,2}$ \\ Bowen $\mathrm{Li}^{3}$ \\ Min Liu ${ }^{1,2}$ \\ Zenan $\mathrm{Hu}^{1,2}$ \\ Yuping Wang ${ }^{1,2}$
}

'Department of Gastroenterology,

The First Hospital of Lanzhou

University, Lanzhou, People's

Republic of China; ${ }^{2}$ Key Laboratory for Gastrointestinal Diseases of

Gansu Province, Lanzhou University, Lanzhou, People's Republic of China;

${ }^{3}$ Department of Pediatrics, The First Hospital of Lanzhou University,

Lanzhou, People's Republic of China

Correspondence: Qian Ren

Department of Gastroenterology,

The First Hospital of Lanzhou University,

No.I Donggang West Road, Chengguan

District, Lanzhou 730000, People's

Republic of China

Email rqian1983@163.com
Background and objective: Many studies have reported that NEK2 is overexpressed in digestive system cancers (DSCs) and is also correlated with patient survival. We performed a meta-analysis to comprehensively evaluate the prognostic role of NEK2 expression in DSCs.

Materials and methods: A comprehensive literature search was performed using PubMed, EMBASE, and Web of Science. Synthesized hazard ratios (HRs) and odds ratios (ORs) with 95\% confidence intervals (CIs) were used to evaluate the influence of NEK2 overexpression on the prognosis and clinicopathological features of patients with DSCs.

Results: A total of 13 studies involving 1,917 patients was included. Overall, patients with high NEK2 expression had poorer overall survival (HR $=1.45 ; 95 \%$ CI: $1.15-1.83 ; P=0.002$ ) and disease-free survival/recurrence-free survival ( $\mathrm{HR}=2.28 ; 95 \% \mathrm{CI}: 1.54-3.37 ; P<0.0001$ ). Furthermore, subgroup analysis also suggested that elevated NEK2 expression was associated with poorer overall survival in patients with hepatocellular carcinoma ( $\mathrm{HR}=1.45 ; 95 \% \mathrm{CI}$ : $1.05-2.00 ; P=0.02)$ and colorectal cancer $(\mathrm{HR}=2.03 ; 95 \% \mathrm{CI}: 1.16-3.54 ; P=0.01)$. Additionally, NEK2 overexpression was also associated with pretreatment serum AFP level (OR $=1.79 ; 95 \%$ CI: $1.23-2.61 ; P<0.01)$ and portal vein thrombosis (OR $=2.74 ; 95 \% \mathrm{CI}: 1.22-6.17 ; P=0.01)$ in hepatocellular carcinoma.

Conclusion: NEK2 might act as a useful prognostic predictor and a potential therapeutic target in DSCs. However, multicenter homogeneous studies with larger sample sizes are needed to further confirm our findings owing to some limitations in our meta-analysis.

Keywords: NEK2, digestive system cancer, prognostic, meta-analysis

\section{Introduction}

Digestive system cancers (DSCs) represent considerable threats to human life and health. ${ }^{1}$ Despite decreasing prevalence of cancer-related mortality during the past 2 decades, the incidence rates of DSCs, including cancers of the esophagus, pancreas, liver, and intestine, continue to increase. ${ }^{2}$ Moreover, hepatocellular carcinoma (HCC) and colorectal cancer (CRC) remain the major medical problems and cause high mortality and heavy economic burdens..$^{3-5}$ Although diagnostic and therapeutic strategies for DSCs have been greatly improved, the prognosis of patients is still unsatisfactory. Clinicopathological characteristics have been widely used as prognostic factors in clinical practice, such as tumor differentiation, size, and number. However, the prognosis of patients with DSCs who have similar clinicopathological characteristics sometimes does not appear to be consistent. Hence, finding additional molecular markers with substantial prognostic value is urgently needed. 
NEK2 belongs to the serine/threonine kinase family and is important for the regulation of centrosome cell cycle, and mitosis. ${ }^{6,7}$ In mitosis, the activation of Nek2 may lead to the phosphorylation of C-Nap1, rootletin, and $\beta$-catenin, which could promote centrosome separation by dissociating these proteins from centrosomes. ${ }^{8-12}$ The increase in NEK2 expression may disturb the management of the mitotic checkpoint, subsequently contributing to the formation of centrosomal abnormalities and monopolar spindles. ${ }^{7,13,14}$ Furthermore, uncontrolled NEK2 activity was involved in rearrangements and abnormal amplification of chromosomes, which leads to chromosome instability (CIN). ${ }^{7,14-17}$ Overall, the biological functions of NEK2 mentioned indicate that the overexpression and aberrant activity of NEK2 might play a key role in tumorigenesis. Recently, several studies reported that NEK2 is overexpressed in various types of cancers, such as CRC breast cancer, pancreatic cancer, and lung adenocarcinoma. ${ }^{18-24}$ In addition, the expression of NEK2 is closely associated with the prognosis and clinicopathological characteristics of $\mathrm{CRC},{ }^{25,26}$ pancreatic ductal adenocarcinoma (PDAC), ${ }^{19}$ and HCC. ${ }^{27}$ However, there were inconsistencies regarding the prognostic role of NEK2 in DSCs, ${ }^{19,28,29}$ which might be partly attributed to the small sample size of any single study exploring the prognostic significance of NEK2. Therefore, we performed a systematic review and meta-analysis to comprehensively evaluate the prognostic value of NEK2 in DSCs by combining the data of multiple relevant studies.

\section{Materials and methods}

To maximize clarity and transparency, this meta-analysis was conducted in accordance with the Preferred Reporting Items for Systematic Reviews and Meta-Analyses (PRISMA) statement issued in 2009. ${ }^{30}$

\section{Literature search strategy and selection criteria}

We comprehensively searched relevant studies assessing the relationship between NEK2 and the prognosis of patients with DSCs in PubMed, EMBASE, and Web of Science. The last search was performed in March 27, 2018. The search terms included: "NEK2 or NIMA related kinase 2" and "cancer or tumor or malignancy or carcinoma or neoplasm."

Studies were included if they met the following eligibility criteria: 1) the associations between NEK2 expression and the prognosis or the clinicopathological characteristics of patients with DSCs were reported and 2) the studies were published in English. On the contrary, the studies would be excluded if they met any of the exclusion criteria: 1) reviews, letters, comments, case reports, animal studies, noncomparative studies, and meeting abstracts; 2) articles with insufficient clinical data; and 3) duplicated articles.

\section{Data extraction}

Data were independently extracted by 2 investigators. The senior investigator resolved incompatibilities between the reviewers in data extraction. The basic information extracted from the literature included the name of first author, publication year, country, cancer type, the number of subjects, gender, tumor stage, detection methods, cutoff scores for NEK2 overexpression, and follow-up. The endpoints of interest included overall survival (OS), disease-free survival (DFS), recurrence-free survival (RFS), and clinicopathologic characteristics.

\section{Quality assessment and statistical analysis}

The quality of eligible articles was assessed using the Newcastle-Ottawa scale (NOS), ${ }^{31}$ which consists of 3 aspects: selection, comparability, and outcome. The score of NOS ranged from 0 to 9 , and articles with scores $>6$ were considered as high quality.

Cochrane Collaboration' s Review Manager 5.3 (Cochrane Collaboration, Oxford, UK) was used to synthesize all the data, and Stata version 12.0 (StataCorp LP, College Station, TX, USA) was employed to perform sensitive analysis, meta-regression analysis, and publication bias assessment. Synthesized hazard ratios (HRs) with 95\% confidence intervals (CIs) were used to describe the prognostic value of NEK2 on survival outcomes in DSCs, and odds ratios (ORs) with 95\% CIs were used to evaluate the relationship between the clinicopathologic characteristics and NEK2 expression. Heterogeneity among the published articles was assessed by Cochran's $Q$ and Higgins $I^{2}$ statistics. The combination of $P<0.05$ and $I^{2}>50 \%$ indicates significant heterogeneity, in which situation data were pooled using a random-effects model. Otherwise, a fixed-effects model was applied to pool the data. In order to minimize the influence of confounding factors and to explore potential source of heterogeneity, subgroup analysis was performed according to country, cancer type, sample size, and detection method. Sensitivity analysis was performed by omitting each individual article step-by-step to test the stability of the meta-analysis results. Furthermore, meta-regression analysis was also used to investigate potential source of heterogeneity. Publication bias was assessed by Begg's funnel plots ${ }^{32}$ and Egger's test. ${ }^{33}$ Symmetrical plots and $P$-value $>0.05$ indicate that there is no significant publication bias across the included studies. 


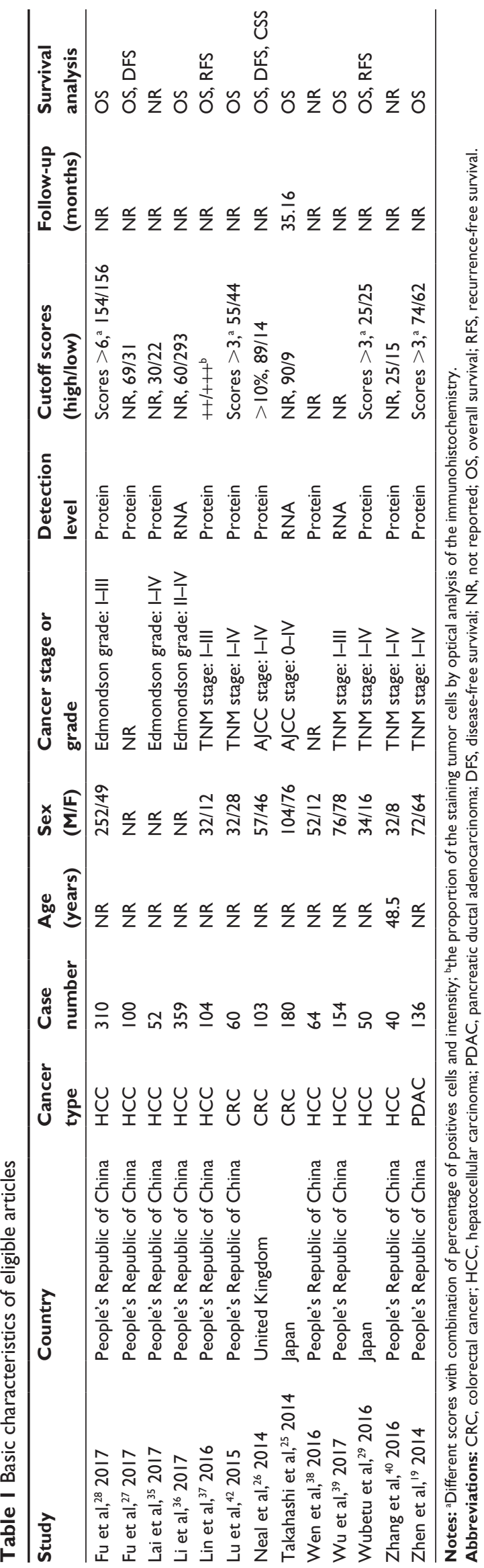

When significant publication bias exists, the trim and fill method was used to estimate the influence of publication bias on the stability of meta-analysis results. ${ }^{34}$

\section{Results}

\section{Search results and study characteristics}

The main characteristics of the eligible, included studies are presented in Table 1. Overall, the initial search yielded 622 publications by retrieving PubMed, EMBASE, and Web of Science entries. After excluding 59 duplicate articles, 563 articles remained for screening of the title and abstract, after which process 517 articles were excluded for being reviews and comments $(n=26)$ and irrelevant studies $(n=491)$. Next, 46 articles were left for full-text screening and, consequently, 33 articles were excluded for being non-DSCs $(\mathrm{n}=4)$, conference abstracts $(\mathrm{n}=13)$, and lacking extractable data $(\mathrm{n}=16)$. Eventually, 13 articles involving 1,917 patients were included in this meta-analysis. ${ }^{25-29,35-42}$ The details of the study search and selection processes are shown in Figure 1.

All of the eligible studies were published in the most recent 5 years (2014-2017). A total of 10 studies were investigated involving the Chinese population, 2 included Japanese individuals, and 1 represented a British study. A total of 9 studies analyzed the relationship between NEK2 and $\mathrm{HCC}, 3$ studies reported the role of NEK2 in CRC, and 1 study referred to PDAC. The expression of NEK2 at the protein level was detected by immunohistochemistry (IHC) in 7 studies, and 2 studies utilized reverse transcription

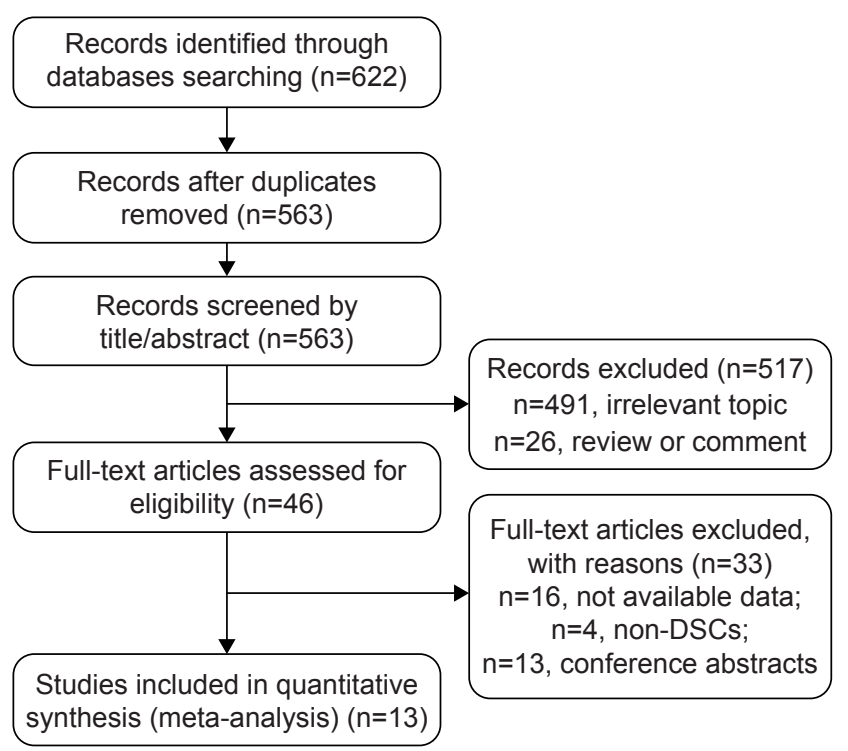

Figure I Flow chart representing the article selection process. Abbreviation: DSC, digestive system cancer. 


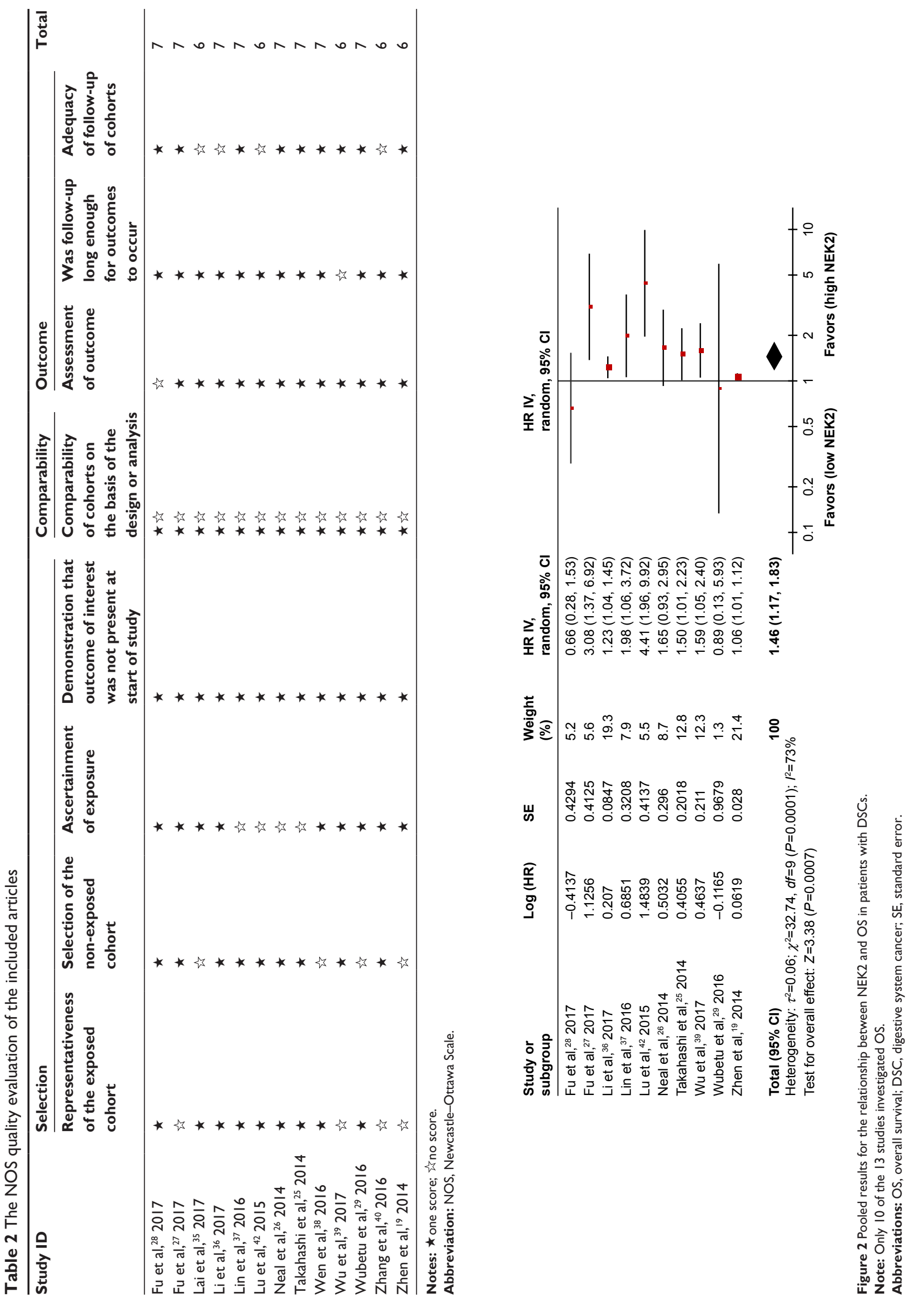




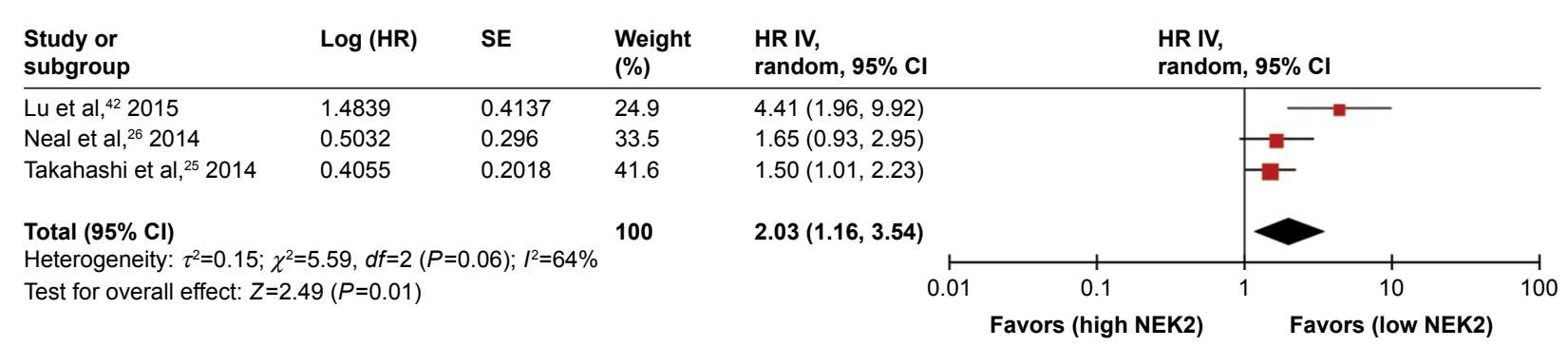

Figure 3 Pooled results for the relationship between NEK2 and OS in CRC patients. Abbreviations: OS, overall survival; CRC, colorectal cancer; SE, standard error.

polymerase chain reaction (RT-qPCR) to describe the expression of NEK2 at the RNA level, and 1 study used TCGA RNASeqV2 database. In addition, the prognostic value of NEK2 in OS was investigated in 10 studies involving 1,556 patients, and associations between NEK2 expression and DFS/RFS were described in 4 studies with 357 patients. Upon quality assessment of the included studies, all of the eligible studies were found to be of high quality and were scored as 6 or more based on NOS values (Table 2).

\section{Prognostic role of NEK2 in digestive system tumors with regard to OS}

A total of 10 studies involving 1,556 patients described the relationship between NEK2 and OS. ${ }^{26,35,36,38-41,43,44,46}$ Since significant heterogeneity among the studies was found $\left(I^{2}=73 \%\right.$, $P<0.01$ ), the randomized effects model was used to calculate the pooled $\mathrm{HR}$ and $95 \% \mathrm{CI}$. Elevated expression of NEK2 was significantly correlated with worse OS (HR $=1.4595 \% \mathrm{CI}$ : $1.15-1.83 ; P=0.002$, Figure 2). Additionally, subgroup analysis was conducted based on cancer type, country, sample size, and detection level. We found that high expression of NEK2 could act as an independent prognostic factor in CRC ( $\mathrm{HR}=2.03$; 95\% CI: $1.16-3.54 ; P=0.01$, Figure 3$)$ and $\mathrm{HCC}(\mathrm{HR}=1.45$; 95\% CI: $1.05-2.00 ; P=0.02$, Figure 4). Furthermore, for the subgroup of country, it was observed that NEK2 was closely correlated with poorer OS in Chinese populations ( $\mathrm{HR}=1.46$; 95\% CI: $1.13-1.90 ; P<0.01)$. Similarly, NEK2 was significantly associated with worse OS in large sample sizes ( $\mathrm{n}>150$ : $\mathrm{HR}=1.30 ; 95 \% \mathrm{CI}: 1.05-1.61 ; P=0.02)$ and in small sample size groups ( $\mathrm{n} \leq 150: \mathrm{HR}=1.90 ; 95 \% \mathrm{CI}: 1.12-3.21 ; P=0.02$ ). For the subgroup involving detection level, we found that patients with high NEK2 RNA and protein expression both had a worse OS (RNA: HR $=1.30 ; 95 \%$ CI: $1.13-1.50 ; P<0.01$; protein: $\mathrm{HR}=1.65 ; 95 \% \mathrm{CI}: 1.03-2.63 ; P=0.04)$. All results of subgroup analysis are shown in Table 3 .

\section{Prognostic role of NEK2 in digestive system tumors in DFS/RFS}

Considering the similarity between DFS and RFS, we merged these data to evaluate the prognostic value of NEK2. Due to the absence of heterogeneity among articles $\left(I^{2}=0 \%, P=0.54\right)$, a fixed-effects model was used to pool the HR values. The results indicated that overexpression of NEK2 was related to significantly worse DFS/RFS (HR $=2.28$; 95\% CI: $1.54-3.37$; $P<0.0001$ ) (Figure 5).

\section{Relationship of NEK2 with clinicopathological characteristics of DSCs} Owing to the differences in clinicopathological features of various cancers, the association of high NEK2 expression

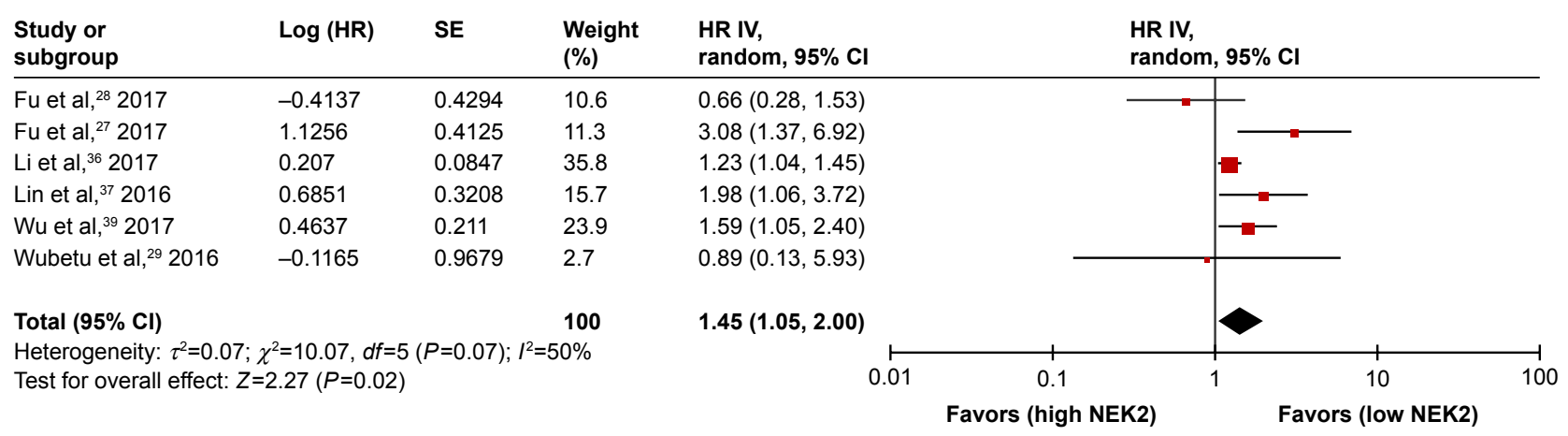

Figure 4 Pooled results for the relationship between NEK2 and OS in liver cancer patients. Note: Only 6 of included studies investigated OS in liver cancer.

Abbreviations: OS, overall survival; SE, standard error. 
Table 3 Results of subgroup analysis of synthesized HRs of OS of patients with NEK2

\begin{tabular}{|c|c|c|c|c|c|c|c|}
\hline \multirow[t]{2}{*}{ Subgroup analysis } & \multirow{2}{*}{$\begin{array}{l}\text { Number } \\
\text { of studies }\end{array}$} & \multirow{2}{*}{$\begin{array}{l}\text { Number } \\
\text { of patients }\end{array}$} & \multirow{2}{*}{$\begin{array}{l}\text { Pooled HR } \\
(95 \% \mathrm{Cl})\end{array}$} & \multirow[t]{2}{*}{$P$-value } & \multicolumn{3}{|c|}{ Heterogeneity } \\
\hline & & & & & $I^{2}(\%)$ & $P$-value & Model \\
\hline \multicolumn{8}{|l|}{ Tumor type } \\
\hline $\mathrm{HCC}$ & 6 & 1,233 & $1.45[1.05,2.00]$ & 0.02 & 50 & 0.07 & Fixed model \\
\hline CRC & 3 & 343 & $2.03[1.16,3.54]$ & 0.01 & 64 & 0.06 & Randomized model \\
\hline PDAC & I & 136 & $1.06[1.00,1.12]$ & 0.05 & - & - & - \\
\hline \multicolumn{8}{|l|}{ Country } \\
\hline People's Republic of China & 7 & $\mathrm{I}, 379$ & $1.46[1.13,1.90]$ & $<0.01$ & 79 & $<0.01$ & Randomized mode \\
\hline United Kingdom & I & 103 & $1.65[0.93,2.95]$ & 0.09 & - & - & - \\
\hline Japan & 2 & 230 & $1.47[1.00,2.16]$ & 0.05 & 0 & 0.60 & Fixed model \\
\hline \multicolumn{8}{|l|}{ Sample size } \\
\hline$>150$ & 4 & $\mathrm{I}, 003$ & $1.30[1.05,1.61]$ & 0.02 & 30 & 0.32 & Fixed model \\
\hline$\leq 150$ & 6 & 709 & $1.90[1.12,3.21]$ & 0.02 & 79 & $<0.01$ & Randomized mode \\
\hline \multicolumn{8}{|l|}{ Detection level } \\
\hline Protein & 7 & 863 & $1.65[1.03,2.63]$ & 0.04 & 76 & $<0.01$ & Randomized mode \\
\hline RNA & 3 & 693 & $1.30[1.13,1.50]$ & $<0.01$ & 0 & 0.40 & Fixed model \\
\hline
\end{tabular}

Abbreviations: OS, overall survival; HCC, hepatocellular carcinoma; CRC, colorectal cancer; PDAC, pancreatic ductal adenocarcinoma.

with clinicopathological characteristics was investigated according to cancer type. As presented in Table 4, NEK2 levels exhibited no obvious correlation with tumor size, differentiation, HBsAg status, hepatic cirrhosis, tumor number and differentiation in HCC, and lymph node metastasis in CRC. However, it was observed that high expression of NEK2 was significantly associated with positive AFP (6 studies, $\mathrm{n}=616,+\mathrm{vs}-$, OR $=1.79 ; 95 \%$ CI: $1.23-2.61 ; P<0.01)$ and the occurrence of portal vein thrombosis (3 studies, $\mathrm{n}=206,+\mathrm{vs}-, \mathrm{OR}=2.74 ; 95 \%$ CI: $1.22-6.17 ; P=0.01)$.

\section{Sensitivity analysis and meta-regression analysis}

Sensitivity analysis was performed to test the robustness of the synthesized results of the effect of NEK2 on OS in DSCs by omitting single studies, stepwise. We found that the pooled HR for OS was not altered significantly when excluding any individual study, suggesting that our synthesized results of the effect of NEK2 on OS were rigorous
(Figure 6). The robustness of the synthesized result for DFS/ RFS was not evaluated by sensitivity analysis because of limited numbers of relevant studies.

Considering the significant heterogeneity for the pooled HR data for OS, meta-regression analysis was further conducted to identify the source of heterogeneity, based on the cancer type, country, sample size, and detection level. However, similar to the results of our subgroup analysis, the outcomes of meta-regression analysis indicated that these factors might not account for the heterogeneity either (Table 5).

\section{Publication bias}

We used Egger's and Begg's tests to evaluate publication bias in the meta-analysis of the influence of NEK2 on OS in DSCs. It was found that Begg's test had a value of 0.79, Egger's test had a value of 0.93 , and the funnel plot was symmetrical, indicating that there was no significant publication bias (Figure 7). Publication bias was not assessed in the

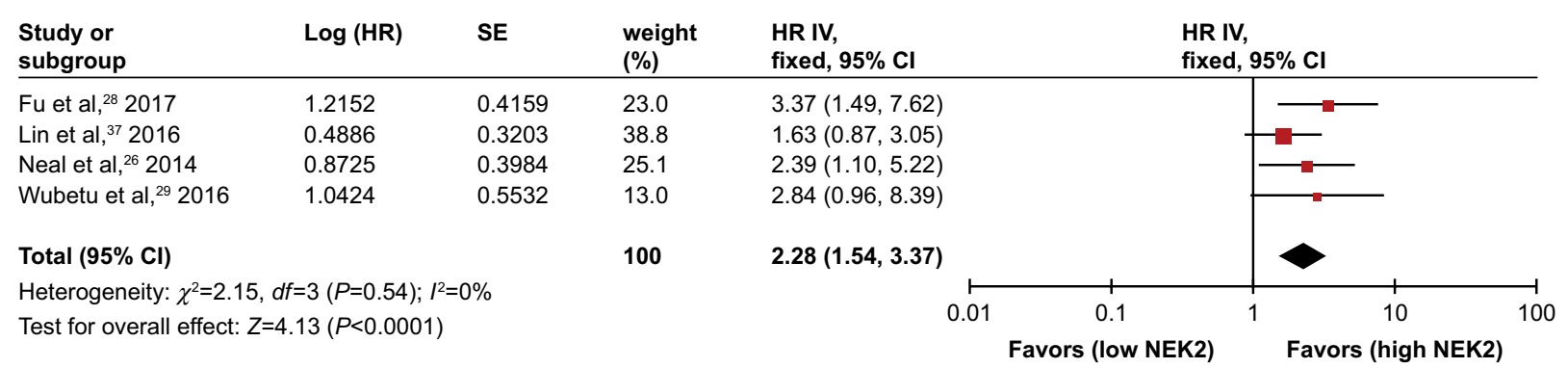

Figure 5 Pooled results for the relationship between NEK2 and DFS/RFS in patients with DSCs. Notes: Only 4 of the 13 studies investigated DFS/RFS.

Abbreviations: DFS/RFS, disease-free survival/recurrence-free survival; DSC, digestive system cancer. 
Table 4 Meta-analysis results of the associations of NEK2 with clinicopathological parameters

\begin{tabular}{|c|c|c|c|c|c|}
\hline \multirow[t]{2}{*}{ Clinicopathological parameter } & \multirow{2}{*}{$\begin{array}{l}\text { Number } \\
\text { of studies }\end{array}$} & \multirow[t]{2}{*}{ OR $(95 \% \mathrm{Cl})$} & \multirow[t]{2}{*}{$P$-value } & \multicolumn{2}{|c|}{ Heterogeneity test } \\
\hline & & & & $I^{2}$ & $P$-value \\
\hline \multicolumn{6}{|l|}{$\mathrm{HCC}$} \\
\hline Tumor size $(\geq 5$ vs $<5 \mathrm{~cm})$ & 6 & $1.72[0.86,3.45]$ & 0.70 & 41 & 0.16 \\
\hline Differentiation (well/moderate vs poor) & 5 & $0.83[0.39,1.73]$ & 0.61 & 65 & 0.02 \\
\hline $\mathrm{HBsAg}(+\mathrm{vs}-)$ & 4 & $0.91[0.58,1.45]$ & 0.70 & 41 & 0.16 \\
\hline Liver cirrhosis (+ vs -) & 4 & $0.90[0.63,1.29]$ & 0.56 & 0.0 & 0.87 \\
\hline Tumor number (multiple vs single) & 4 & $1.14[0.72,1.80]$ & 0.57 & 0.0 & 0.68 \\
\hline $\operatorname{AFP}(+$ vs -$)$ & 6 & $1.79[1.23,2.61]$ & $<0.01$ & 7.4 & 0.37 \\
\hline Portal vein thrombosis (+ vs -) & 3 & $2.74[1.22,6.17]$ & 0.01 & 40 & 0.19 \\
\hline \multicolumn{6}{|l|}{ CRC } \\
\hline Tumor differentiation (moderate-well vs poor) & 3 & $0.5 \mathrm{I}[0.24,1.08]$ & 0.08 & 10 & 0.33 \\
\hline Lymph node metastasis (+ vs -) & 3 & $1.74[0.28,10.69]$ & 0.55 & 87 & $<0.01$ \\
\hline
\end{tabular}

Abbreviations: AFP, alpha fetoprotein; HCC, hepatocellular carcinoma; CRC, colorectal cancer.

meta-analysis of the influence of NEK2 on DFS/RFS owing to the limited number of relevant studies.

\section{Discussion}

It is believed that abnormal cell cycle regulation is important for the occurrence and development of cancers, and NEK2 might play a crucial role in regulating cell cycle via promoting the separation of centrosome in the process of cell division and orchestrating the composition and function of centrosomes..$^{13,43,44}$ Furthermore, several studies reported that NEK2 is overexpressed in various cancers and was related to unfavorable prognosis and clinicopathological characteristics, ${ }^{18-24}$ indicating the involvement of NEK2 in tumorigenesis. However, the conclusions were inconsistent with respect to the prognostic role of NEK2 in DSCs. Therefore, the current meta-analysis was performed to comprehensively evaluate the influence of NEK2 overexpression

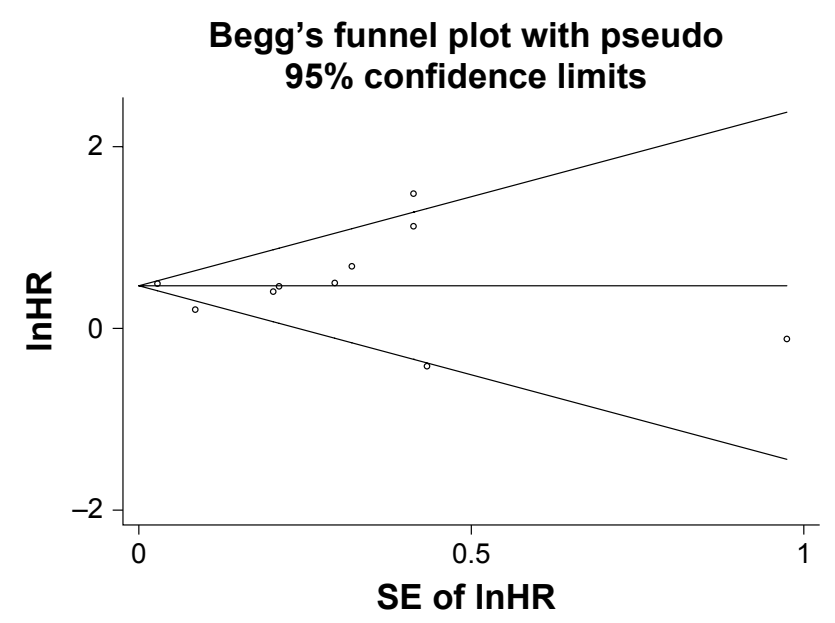

Figure 6 Sensitive analysis of the influence of individual studies on the pooled HR of OS.

Abbreviation: SE, standard error. on the prognosis and clinicopathological characteristics of patients with DSCs.

In the present study, we comprehensively analyzed the survival data of 1,917 patients from 13 studies and found that overexpression of NEK2 was significantly correlated with worse OS and DFS in patients with DSCs. Considering that some confounding factors might affect the reliability of our synthesized results, subgroup analysis and meta-regression were conducted according to several potential confounding factors, such as cancer type, country, sample size, and detection level. From the results, we observed that CRC and HCC patients with elevated NEK2 expression had a poorer survival prognosis. Moreover, with different sample sizes and detection levels, the overexpression of NEK2 consistently acted as an indicator of worse OS in patients with DSCs. Additionally, we also investigated the relationship between NEK2 expression and clinicopathological characteristics in patients with DSCs. Our results indicated that high expression of NEK2 was significantly associated with serum positive AFP levels and portal vein thrombosis in $\mathrm{HCC}$, which might partly explain the role of NEK2 overexpression as an indicator of poorer OS in HCC.

Table 5 The influence of study characteristics on heterogeneity assessed by meta-regression

\begin{tabular}{|c|c|c|c|}
\hline Study characteristic & $P$-value & $\begin{array}{l}\text { Regression } \\
\text { coefficient }\end{array}$ & $95 \% \mathrm{Cl}$ \\
\hline $\begin{array}{l}\text { Cancer type (HCC vs CRC vs } \\
\text { PDAC) }\end{array}$ & 0.601 & 1.09 & $(0.74-1.62)$ \\
\hline $\begin{array}{l}\text { Country (People's Republic of } \\
\text { China vs Japan vs United Kingdom) }\end{array}$ & 0.89 & 0.97 & $(0.59-1.59)$ \\
\hline Sample size $(>\mid 50$ vs $\leq \mid 50)$ & 0.09 & 1.43 & $(0.93-2.20)$ \\
\hline Detection level (protein vs RNA) & 0.24 & 0.79 & $(0.5 I-I .22)$ \\
\hline
\end{tabular}

Abbreviations: HCC, hepatocellular carcinoma; CRC, colorectal cancer; PDAC, pancreatic ductal adenocarcinoma. 
Meta-analysis random-effects estimates (exponential form) study ommited

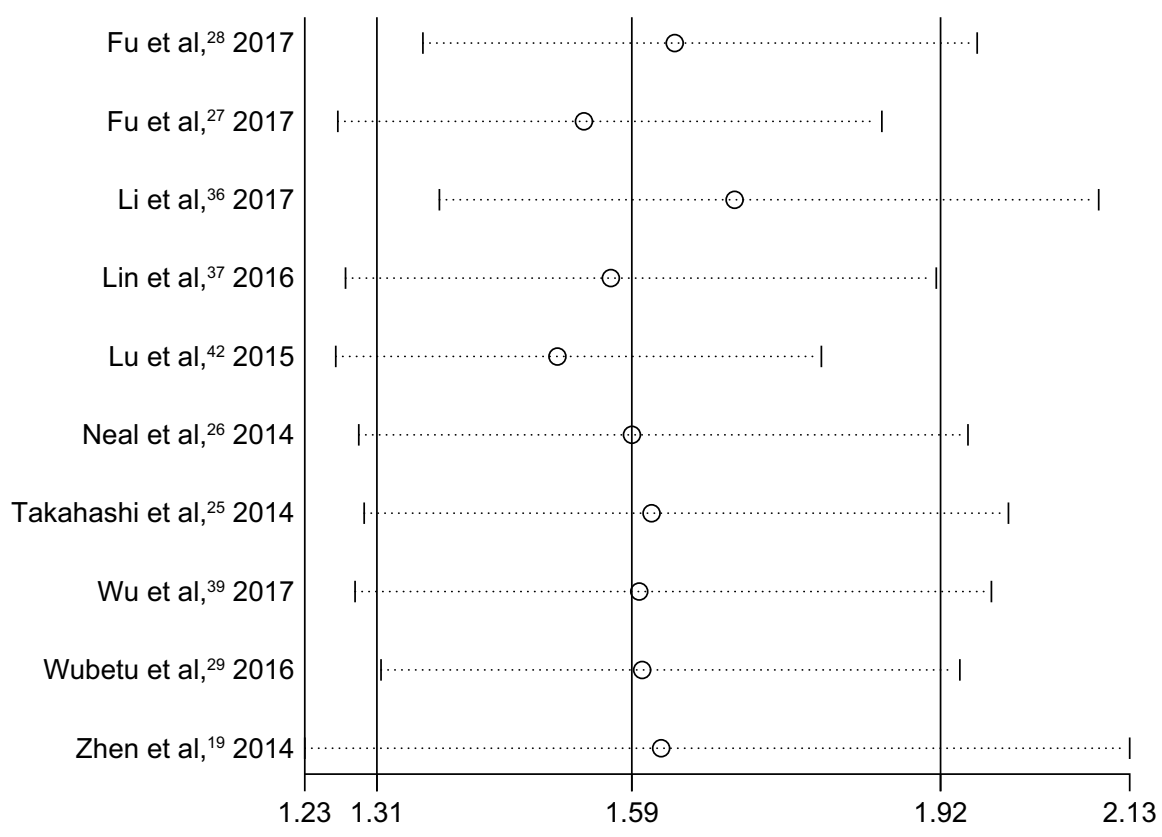

Figure 7 Begg's funnel plot for potential publication bias involving eligible studies reporting OS Abbreviation: OS, overall survival.

To date, several mechanisms have been reported to be responsible for the oncogenic roles of NEK2, which might explain the inverse association between NEK2 expression and prognosis of patients with DSCs. Elevated expression of NEK2 and its homologs could lead to abnormal aggregation of centrosomes and splitting of chromatin, and finally dramatic mitotic progress, which may contribute to the aberrant proliferation of cells. ${ }^{45,46}$ It has been reported that NEK2 overexpression could enhance tumor progression by involving the activation of the PI3K/AKT pathway. ${ }^{16,46}$ For instance, Li et al ${ }^{36}$ found that overexpression of NEK2 could promote the invasion and metastasis of $\mathrm{HCC}$ by activating AKT signaling and upregulating the expression of MMP-2. Additionally, NEK2 overexpression was also reported to abnormally activate the MAPK and Wnt/ $\beta$-catenin pathways, which contributed to the proliferation, invasion, and migration of cancer cells. ${ }^{26,37,40}$ More importantly, Lin et al ${ }^{37}$ and $\mathrm{Fu}$ et $\mathrm{al}^{27}$ found that deletion of NEK2 could significantly reduce stem-like properties, proliferation, colony formation, migration, and invasion in in vivo tumors formed by HCC cell lines, indicating that NEK2 has certain biological functions that driver genes specifically have. Therefore, we speculated that NEK2 might act as a driver gene and exhibit the potential to be a useful molecular target in $\mathrm{HCC}$ and in other digestive system tumors. Of course, more rigorous studies are required to determine whether NEK2 is a driver or passenger gene in digestive system tumors.
There were some limitations to our meta-analysis, which should be considered when interpreting our results. First, all the eligible studies were retrospectively designed, which inevitably had a bias risk and influenced the dependability of our synthesized results. Second, most of the studies were conducted in Asian populations, so our meta-analysis might not represent the worldwide population. Third, although our subgroup analysis and meta-regression indicated that cancer type, country, detection method, and cutoff value might not be responsible for the source of heterogeneity, the reliability of our pooled results were still challenged by significant heterogeneity. Thus, homogeneous studies are needed to further investigate the prognostic role of NEK2 in patients with DSCs. Fourth, only 4 eligible studies with small sample sizes provided data on DFS/RFS for our meta-analysis, which might be insufficient to draw a robust conclusion in this regard. Last but not least, in this meta-analysis, some survival data were manually extracted from Kaplan-Meier curves, which also caused unavoidable bias and heterogeneity.

\section{Conclusion}

In summary, this study demonstrated that NEK2 could act as a useful prognostic indicator and as a promising therapeutic target in DSCs. However, considering the limitations mentioned above, multicenter homogeneous studies with lager sample sizes are needed to further confirm our findings. Meanwhile, the relevant mechanisms underlying 
the prognostic roles of NEK2 in digestive system tumors, including NEK2 interaction with other genes or molecules and tumor-associated signaling pathways, should also be further explored in the future.

\section{Acknowledgment}

We thank Youth Science Foundation of Gansu Province (145RJYA287), Science Foundation of Gansu Province (18JR3RA352), and the First Hospital of Lanzhou University Foundation (ldyyynqn201201) for supporting this study.

\section{Disclosure}

The authors report no conflicts of interest in this work.

\section{References}

1. Chen WQ, Hu L, Chen GX, Deng HX. Role of microRNA-7 in digestive system malignancy. World J Gastrointest Oncol. 2016;8(1): $121-127$.

2. Siegel RL, Miller KD, Jemal A. Cancer Statistics, 2017. CA Cancer J Clin. 2017;67(1):7-30

3. Wang C, Zhang J, Cai M, et al. DBGC: A database of human gastric cancer. PLoS One. 2015;10(11):e0142591.

4. Shim JH, Jun MJ, Han S, et al. Prognostic nomograms for prediction of recurrence and survival after curative liver resection for hepatocellular carcinoma. Ann Surg. 2015;261(5):939-946.

5. Chan AW, Chan SL, Wong GL, et al. Prognostic Nutritional Index (PNI) predicts tumor recurrence of very early/early stage hepatocellular carcinoma after surgical resection. Ann Surg Oncol. 2015;22(13): 4138-4148.

6. Fry AM. The Nek2 protein kinase: a novel regulator of centrosome structure. Oncogene. 2002;21(40):6184-6194

7. Fletcher L, Cerniglia GJ, Nigg EA, Yend TJ, Muschel RJ. Inhibition of centrosome separation after DNA damage: a role for Nek2. Radiat Res. 2004;162(2):128-135.

8. Bahe S, Stierhof YD, Wilkinson CJ, Leiss F, Nigg EA. Rootletin forms centriole-associated filaments and functions in centrosome cohesion. J Cell Biol. 2005;171(1):27-33.

9. Bahmanyar S, Kaplan DD, Deluca JG, et al. beta-Catenin is a Nek2 substrate involved in centrosome separation. Genes Dev. 2008;22(1): 91-105.

10. Fry AM, Mayor T, Meraldi P, Stierhof YD, Tanaka K, Nigg EA. C-Nap1, a novel centrosomal coiled-coil protein and candidate substrate of the cell cycle-regulated protein kinase Nek2. J Cell Biol. 1998; 141(7):1563-1574.

11. Mayor T, Hacker U, Stierhof YD, Nigg EA. The mechanism regulating the dissociation of the centrosomal protein C-Nap1 from mitotic spindle poles. J Cell Sci. 2002;115(Pt 16):3275-3284.

12. Yang J, Adamian M, Li T. Rootletin interacts with C-Nap1 and may function as a physical linker between the pair of centrioles/basal bodies in cells. Mol Biol Cell. 2006;17(2):1033-1040.

13. Fry AM, Meraldi P, Nigg EA. A centrosomal function for the human Nek2 protein kinase, a member of the NIMA family of cell cycle regulators. EMBO J. 1998;17(2):470-481.

14. Prigent C, Glover DM, Giet R. Drosophila Nek2 protein kinase knockdown leads to centrosome maturation defects while overexpression causes centrosome fragmentation and cytokinesis failure. Exp Cell Res. 2005;303(1):1-13.

15. Sen S. Aneuploidy and cancer. Curr Opin Oncol. 2000;12(1):82-88.

16. Zhou W, Yang Y, Xia J, et al. NEK2 induces drug resistance mainly through activation of efflux drug pumps and is associated with poor prognosis in myeloma and other cancers. Cancer Cell. 2013;23(1): $48-62$.
17. Cappello P, Blaser H, Gorrini C, et al. Role of Nek2 on centrosome duplication and aneuploidy in breast cancer cells. Oncogene. 2014;33(18): 2375-2384.

18. Hayward DG, Clarke RB, Faragher AJ, Pillai MR, Hagan IM, Fry AM. The centrosomal kinase Nek2 displays elevated levels of protein expression in human breast cancer. Cancer Res. 2004;64(20):7370-7376.

19. Ning Z, Wang A, Liang J, et al. Abnormal expression of Nek2 in pancreatic ductal adenocarcinoma: a novel marker for prognosis. Int $J$ Clin Exp Pathol. 2014;7(5):2462-2469.

20. Landi MT, Dracheva T, Rotunno M, et al. Gene expression signature of cigarette smoking and its role in lung adenocarcinoma development and survival. PLoS One. 2008;3(2):e1651.

21. de Vos S, Hofmann WK, Grogan TM, et al. Gene expression profile of serial samples of transformed B-cell lymphomas. Lab Invest. 2003;83(2):271-285.

22. Tsunoda N, Kokuryo T, Oda K, et al. Nek2 as a novel molecular target for the treatment of breast carcinoma. Cancer Sci. 2009;100(1):111-116.

23. Kokuryo T, Senga T, Yokoyama Y, Nagino M, Nimura Y, Hamaguchi M. Nek2 as an effective target for inhibition of tumorigenic growth and peritoneal dissemination of cholangiocarcinoma. Cancer Res. 2007; 67(20):9637-9642.

24. Suzuki K, Kokuryo T, Senga T, Yokoyama Y, Nagino M, Hamaguchi M. Novel combination treatment for colorectal cancer using Nek2 siRNA and cisplatin. Cancer Sci. 2010;101(5):1163-1169.

25. Takahashi Y, Iwaya T, Sawada G, et al. Up-regulation of NEK2 by microRNA-128 methylation is associated with poor prognosis in colorectal cancer. Ann Surg Oncol. 2014;21(1):205-212.

26. Neal CP, Fry AM, Moreman C, et al. Overexpression of the Nek2 kinase in colorectal cancer correlates with beta-catenin relocalization and shortened cancer-specific survival. J Surg Oncol. 2014;110(7): 828-838.

27. Fu SJ, Chen J, Ji F, et al. MiR-486-5p negatively regulates oncogenic NEK2 in hepatocellular carcinoma. Oncotarget. 2017;8(32): 2948-52959.

28. Fu L, Liu S, Wang H, et al. Low expression of NEK2 is associated with hepatocellular carcinoma progression and poor prognosis. Cancer Biomark. 2017;20(1):101-106.

29. Wubetu GY, Morine Y, Teraoku H, et al. High NEK2 expression is a predictor of tumor recurrence in hepatocellular carcinoma patients after hepatectomy. Anticancer Res. 2016;36(2):757-762.

30. Moher D, Liberati A, Tetzlaff J, Altman DG; PRISMA Group. Preferred reporting items for systematic reviews and meta-analyses: the PRISMA statement. Int J Surg. 2010;8(5):336-341.

31. Stang A. Critical evaluation of the Newcastle-Ottawa scale for the assessment of the quality of nonrandomized studies in meta-analyses. Eur J Epidemiol. 2010;25(9):603-605.

32. Begg CB, Mazumdar M. Operating characteristics of a rank correlation test for publication bias. Biometrics. 1994;50(4):1088-1101.

33. Egger M, Davey Smith G, Schneider M, Minder C. Bias in meta-analysis detected by a simple, graphical test. BMJ. 1997;315(7109):629-634.

34. Duval S, Tweedie R. Trim and fill: A simple funnel-plot-based method of testing and adjusting for publication bias in meta-analysis. Biometrics. 2000;56(2):455-463.

35. Lai XB, Nie YQ, Huang HL, et al. NIMA-related kinase 2 regulates hepatocellular carcinoma cell growth and proliferation. Oncol Lett. 2017;13(3):1587-1594.

36. Li G, Zhong Y, Shen Q, et al. NEK2 serves as a prognostic biomarker for hepatocellular carcinoma. Int J Oncol. 2017;50(2):405-413.

37. Lin $\mathrm{S}$, Zhou $\mathrm{S}$, Jiang $\mathrm{S}$, et al. NEK2 regulates stem-like properties and predicts poor prognosis in hepatocellular carcinoma. Oncol Rep. 2016;36(2):853-862.

38. Wen S, Liu Y, Yang M, Yang K, Huang J, Feng D. Increased NEK2 in hepatocellular carcinoma promotes cancer progression and drug resistance by promoting PP1/Akt and Wnt activation. Oncol Rep. 2016; 36(4):2193-2199.

39. Wu SM, Lin SL, Lee KY, et al. Hepatoma cell functions modulated by NEK2 are associated with liver cancer progression. Int J Cancer. 2017;140(7):1581-1596. 
40. Zhang MX, Xu XM, Zhang P, et al. Effect of silencing NEK2 on biological behaviors of HepG2 in human hepatoma cells and MAPK signal pathway. Tumour Biol. 2016;37(2):2023-2035.

41. Jemal A, Simard EP, Dorell C, et al. Annual Report to the Nation on the Status of Cancer, 1975-2009, featuring the burden and trends in human papillomavirus (HPV)-associated cancers and HPV vaccination coverage levels. J Natl Cancer Inst. 2013;105(3):175-201.

42. Lu L, Zhai X, Yuan R. Clinical significance and prognostic value of Nek2 protein expression in colon cancer. Int J Clin Exp Pathol. 2015; 8(11):15467-15473.

43. Schultz SJ, Fry AM, Sütterlin C, Ried T, Nigg EA. Cell cycle-dependent expression of Nek2, a novel human protein kinase related to the NIMA mitotic regulator of Aspergillus nidulans. Cell Growth Differ. 1994; 5(6):625-635.
44. Uto K, Sagata N. Nek2B, a novel maternal form of Nek2 kinase, is essential for the assembly or maintenance of centrosomes in early Xenopus embryos. EMBO J. 2000;19(8):1816-1826.

45. Hayward DG, Fry AM. Nek2 kinase in chromosome instability and cancer. Cancer Lett. 2006;237(2):155-166.

46. Weiss MM, Kuipers EJ, Postma C, et al. Genomic alterations in primary gastric adenocarcinomas correlate with clinicopathological characteristics and survival. Cell Oncol. 2004;26(5-6):307-317.

\section{Publish your work in this journal}

OncoTargets and Therapy is an international, peer-reviewed, open access journal focusing on the pathological basis of all cancers, potential targets for therapy and treatment protocols employed to improve the management of cancer patients. The journal also focuses on the impact of management programs and new therapeutic agents and protocols on

\section{Dovepress}

patient perspectives such as quality of life, adherence and satisfaction. The manuscript management system is completely online and includes a very quick and fair peer-review system, which is all easy to use. Visit http://www.dovepress.com/testimonials.php to read real quotes from published authors. 